\title{
PILOT: Introducing Transformers for Probabilistic Sound Event Localization
}

\author{
Christopher Schymura ${ }^{1}$, Benedikt Bönninghoff ${ }^{1}$, Tsubasa Ochiai ${ }^{2}$, Marc Delcroix ${ }^{2}$, \\ Keisuke Kinoshita ${ }^{2}$, Tomohiro Nakatani ${ }^{2}$, Shoko Araki ${ }^{2}$, Dorothea Kolossa ${ }^{1}$ \\ ${ }^{1}$ Cognitive Signal Processing Group, Ruhr University Bochum, Bochum, Germany \\ ${ }^{2}$ NTT Communications Science Laboratories, NTT Corporation, Kyoto, Japan \\ \{christopher.schymura, benedikt.boenninghoff, dorothea.kolossa\}@rub.de \\ \{tsubasa.ochiai.ah, keisuke.kinoshita.mb, shoko.araki.pu\}@hco.ntt.co.jp \\ \{marc.delcroix, tnak\}@ieee.org
}

\begin{abstract}
Sound event localization aims at estimating the positions of sound sources in the environment with respect to an acoustic receiver (e.g. a microphone array). Recent advances in this domain most prominently focused on utilizing deep recurrent neural networks. Inspired by the success of transformer architectures as a suitable alternative to classical recurrent neural networks, this paper introduces a novel transformer-based sound event localization framework, where temporal dependencies in the received multi-channel audio signals are captured via self-attention mechanisms. Additionally, the estimated sound event positions are represented as multivariate Gaussian variables, yielding an additional notion of uncertainty, which many previously proposed deep learning-based systems designed for this application do not provide. The framework is evaluated on three publicly available multi-source sound event localization datasets and compared against state-of-the-art methods in terms of localization error and event detection accuracy. It outperforms all competing systems on all datasets with statistical significant differences in performance.

Index Terms: Audio signal processing, self attention, probabilistic deep learning, sound event localization, transformer
\end{abstract}

\section{Introduction}

The ability of a computational system to locate sounds in the environment, often termed sound event localization (SEL), enables a variety of technical applications. In many cases, a sound event refers to a speech source, which is important in applications such as speech enhancement [1,2], speaker and meeting diarization [3] 4], automatic speech recognition [5] and many others. However, a sound event can also be associated with a broader spectrum of acoustic sources, enabling the application of SEL in domains like industrial monitoring [6] and environmental traffic analysis [7]. Specific challenges of SEL include acoustic disturbances caused by background noise and reverberation, as well as the potential temporal overlap of simultaneously active acoustic sources.

These challenges have been addressed by classical SEL systems primarily based on parametric approaches [8-10], as well as by more recently introduced deep learning frameworks [11, 14]. Regardless of the specific architectural considerations, many SEL systems incorporate temporal context into the estimation process. While classical methods usually rely on recursive Bayesian estimation techniques [15 16], modern systems make use of recurrent structures like long-short term memory (LSTM) networks [17]. However, conventional neural network-based systems lack the ability to associate uncertainty with the estimated sound event locations; a feature that tradi-
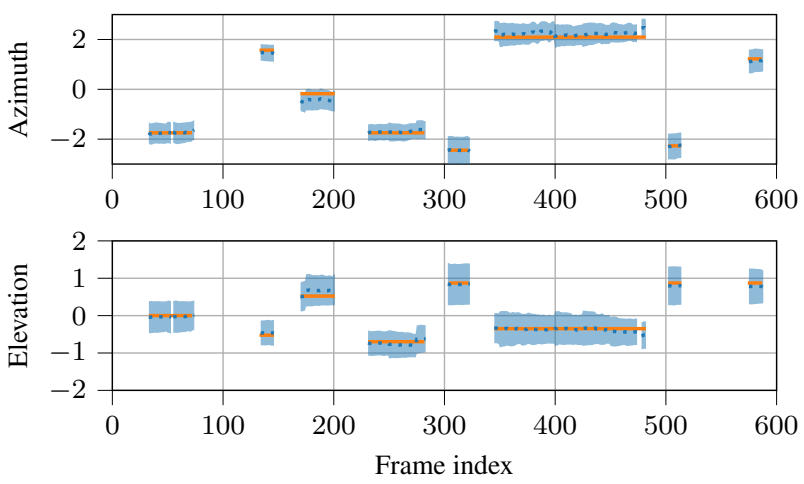

Figure 1: Azimuth and elevation estimates (blue dotted lines) in radians, provided by the proposed PILOT framework. The solid orange lines show ground-truth sound event direction-ofarrivals (DoAs). The estimates provided by PILOT are associated with uncertainty measures of the underlying Gaussian model, depicted as shaded blue regions.

tional Bayesian approaches naturally provide. Additionally, recurrent neural networks (RNNs) are often struggle to learn longterm dependencies within time-series data [18].

This paper proposes a novel deep learning framework for SEL that adopts a transformer-based architecture [19] with a probabilistic representation of sound event locations. Therefore, it will be abbreviated as Probabilistic Localization of Sounds with $\underline{T}$ ransformers (PILOT). Following the design philosophy of the transformer, the proposed model provides a conventional feed-forward signal flow and handles temporal dependencies via self-attention mechanisms. The probabilistic output is based on a differentiable linear Gaussian system (LGS) |20 Chap. 4] model that is adopted in this work to handle multisource SEL scenarios. Fig. 11 depicts exemplary trajectories of DoA estimates provided by PILOT, which illustrate the models capability of associating uncertainty measures with the corresponding predictions.

\section{Related work}

Deep learning-based systems for SEL most prominently rely on CNNs, RNNs or a combination of both. For instance, the model proposed in [11] uses a CNN-based feature extraction, which operates on the STFT spectrum to predict the DoA of a sound event. A similar approach was introduced in [13], where the CNN uses the STFT representation to generate a spatial pseudo spectrum, resembling the DoA spectrum obtained by the multiple signal classification (MUSIC) algorithm. An extension towards handling sound event localization and de- 


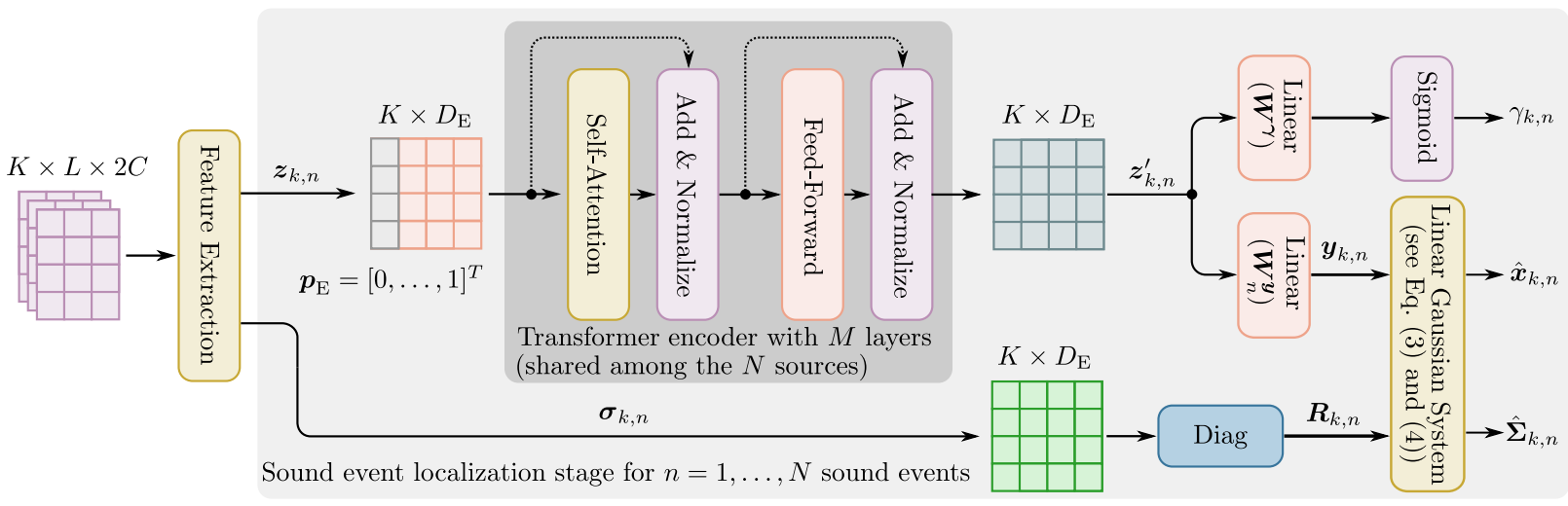

Figure 2: Overview of the PILOT model. An input tensor of the short-time Fourier transform (STFT) representation with K time frames, $L$ frequency bins and $2 C$ channels (containing both magnitude and phase components), is fed into a convolutional neural network $(C N N)$-based feature extraction stage. Positional encodings are attached to the extracted features, resulting in a $D_{\mathrm{E}-\text { dimensional }}$ intermediate representation, which serves as input to the transformer network. The output is then further processed, yielding indicator variables on sound event activity $\gamma_{k, n}$ and their corresponding locations. The latter are represented as multivariate Gaussians with mean $\hat{\boldsymbol{x}}_{k, n}$ and covariance matrix $\hat{\boldsymbol{\Sigma}}_{k, n}$ for each of the $n=1, \ldots, N$ sound events that can be assessed by the model.

tection (SELD) was proposed in [14]. Herein, the task focuses on estimating the association of the sound event type, jointly with its position. The proposed system solved this task via a combination of CNN-based feature extraction and $\mathrm{RNN}$ based estimation of spatial locations and classification of event types. A related system for SEL was proposed in [21], which adopted the CNN-based feature extraction, but exchanged the RNN stage with an attention-based sequence-to-sequence architecture [22]. Additionally, approaches to joining deep learning with classical probabilistic models have recently gained attention. Notable examples are the backprop Kalman filter (KF) [23], the differentiable particle filter [24] and recurrent Kalman networks [25]. Related to the task of SEL, a KF-based deep learning framework was proposed in [26] for audiovisual speaker tracking. Other probabilistic models to tackle SEL are described in, e.g., [27, 28].

\section{System description}

The framework proposed in this work is composed of three individual stages: a CNN-based feature extraction stage, a transformer-based encoder to handle temporal dependencies in the audio signals and a probabilistic output stage that indicates active sound events and their respective spatial locations. An overview of the complete system is depicted in Fig. 2

\subsection{Feature extraction}

The CNN-based feature extractor from SELDNet [14] is utilized in this work. An acoustic waveform with $C=4$ channels is divided into non-overlapping chunks of $500 \mathrm{~ms}$ duration. An STFT with a 2048-point Hamming window, a frame length of $40 \mathrm{~ms}$ and $20 \mathrm{~ms}$ shift, is performed on each chunk. Magnitude and phase of the complex spectrum are concatenated to an input tensor of dimension $K \times L \times 2 C$, where $K=25$ is the number of frames within one chunk and $L=1024$ is the number of non-redundant frequency bins.

A three-layer $\mathrm{CNN}$ is employed to derive features from the STFT representation. Each layer contains 64 filters with kernel size $3 \times 3$, followed by batch normalization, rectified linear units (ReLUs) and max-pooling. The latter has a kernel size of $1 \times 8$ in the first two layers and $1 \times 2$ in the third layer. The third layer output is flattened and fed into a fully-connected network with three layers, intermediate hidden dimensions of 128 and ReLUs as hidden activations. The output layer of this network is composed of two segments: The first segment with linear activations returns feature vectors $\boldsymbol{z}_{k, n} \in \mathbb{R}^{D \mathrm{~F}}$. The second segment produces estimates of the observation noise variances $\sigma_{k, n} \in \mathbb{R}^{D_{\mathrm{E}}}$ with $D_{\mathrm{E}}=D_{\mathrm{F}}+1$, using exponential non-linearities. Both segments produce these outputs for each of the $n=1, \ldots, N$ sound events, where $N$ denotes the maximum number of sound events that the system can handle simultaneously.

\subsection{Transformer network for handling temporal context}

A transformer encoder as introduced in [19] is the primary building block of the intermediate stage. Each sequence of feature vectors $\left\{\boldsymbol{z}_{k, n}\right\}_{k=1}^{K}$ is concatenated with a positional encoding vector $\boldsymbol{p}_{\mathrm{E}}=\left[\begin{array}{lll}p_{\mathrm{E}, 1} & \cdots & p_{\mathrm{E}, K}\end{array}\right]^{\mathrm{T}} \in \mathbb{R}^{K}$ with elements $p_{\mathrm{E}, k}=\frac{1}{K-1}(k-1)$, yielding a $D_{\mathrm{E}}$ dimensional tensor, as depicted in Fig. 22 This approach to encode information about the temporal order differs from the positional encodings described in [19]. Initial experiments have shown that a linearly increasing vector resulted in superior performance over positional encodings based on trigonometric functions.

The transformer encoder itself follows a conventional architecture with $M=3$ layers. Each layer incorporates a selfattention network with 4 heads, followed by layer normalization, a fully-connected feed-forward network with an intermediate dimension of 1024 and another layer normalization stage at the output. Both self-attention and feed-forward networks can be bypassed by additional skip connections, exactly following the specifications outlined in [19]. The transformer output has the same dimensions as the input and will be denoted as $\left\{\boldsymbol{z}_{k, n}^{\prime}\right\}_{k=1}^{K}$, serving as input to the subsequent localization output stage of the PILOT model.

\subsection{Probabilistic localization output stage}

This stage is based on the ADRENALINE system proposed in [21]. It is composed of two components: a vector $\gamma_{k} \in \mathbb{R}^{N}$ with elements $\gamma_{k, n} \in[0,1]$, indicating sound event activity, where $N$ is the maximum number of sound events that the system can handle simultaneously. This vector is obtained as 
$\boldsymbol{\gamma}_{k}=\sigma\left(\boldsymbol{W}^{\boldsymbol{\gamma}} \boldsymbol{z}_{k}^{\prime}\right)$, where $\boldsymbol{W}^{\boldsymbol{\gamma}} \in \mathbb{R}^{N \times D_{\mathrm{E}}}$ is a weight matrix and $\sigma(\cdot)$ denotes the sigmoid function. The spatial position of each sound event is encoded as a multivariate Gaussian variable with mean $\hat{\boldsymbol{x}}_{k, n}$ and covariance matrix $\hat{\boldsymbol{\Sigma}}_{k, n}$. The spatial coordinates are expressed via a DoA representation $\hat{\boldsymbol{x}}_{k, n}=\left[\begin{array}{ll}\hat{\phi}_{k, n} & \hat{\vartheta}_{k, n}\end{array}\right]^{\mathrm{T}}$ with azimuth and elevation angles $\hat{\phi}_{k, n}$ and $\hat{\vartheta}_{k, n}$, respectively.

A prominent advantage of the transformer-based model compared to RNNs is its handling of temporal dependencies in sequence data without recurrent structures. Therefore, using recursive Bayesian estimation techniques in the output stage would render this advantage void. Hence, a LGS is used here, which encodes the individual sound event DoAs as

$$
\begin{aligned}
p\left(\boldsymbol{x}_{k, n}\right) & =\mathcal{N}\left(\boldsymbol{x}_{k, n} \mid \boldsymbol{\mu}_{n}, \boldsymbol{\Sigma}_{n}\right), \\
p\left(\boldsymbol{y}_{k, n} \mid \boldsymbol{x}_{k, n}\right) & =\mathcal{N}\left(\boldsymbol{y}_{k, n} \mid \boldsymbol{C} \boldsymbol{x}_{k, n}+\boldsymbol{b}, \boldsymbol{R}_{k, n}\right),
\end{aligned}
$$

where $\boldsymbol{x}_{k, n}=\left[\begin{array}{ll}\phi_{k, n} & \vartheta_{k, n}\end{array}\right]^{\mathrm{T}}$ is the DoA vector corresponding to the $n$-th sound event. A multivariate Gaussian prior with mean $\boldsymbol{\mu}_{n} \in \mathbb{R}^{2}$ and covariance matrix $\boldsymbol{\Sigma}_{n} \in \mathbb{R}^{2 \times 2}$ is imposed on each DoA vector in Eq. (1). Eq. (2) of the LGS represents a Gaussian observation model, parameterized by an observation matrix $\boldsymbol{C} \in \mathbb{R}^{D_{\mathrm{E}} \times 2}$, bias term $\boldsymbol{b} \in \mathbb{R}^{D_{\mathrm{E}}}$ and observation noise covariance matrix $\boldsymbol{R}_{k, n}=\operatorname{diag}\left(\boldsymbol{\sigma}_{k, n}\right)$. The observation vector $\boldsymbol{y}_{k, n}$ for the $n$-th source is obtained via a linear transformation $\boldsymbol{y}_{k, n}=\boldsymbol{W}_{n}^{\boldsymbol{y}} \boldsymbol{z}_{k, n}^{\prime}$ with weights $\boldsymbol{W}_{n}^{\boldsymbol{y}} \in \mathbb{R}^{D_{\mathrm{E}} \times D_{\mathrm{E}}}$.

An inference scheme to obtain the posterior distribution $p\left(\boldsymbol{x}_{k, n} \mid \boldsymbol{y}_{k, n}\right) \propto p\left(\boldsymbol{y}_{k, n} \mid \boldsymbol{x}_{k, n}\right) p\left(\boldsymbol{x}_{k, n}\right)$ can be derived by computing the first and second derivatives of the log-posterior using Eqs. (1) and (2). Setting the first derivative to zero and solving for $\hat{\boldsymbol{x}}_{k, n}$ yields the posterior mean

$$
\hat{\boldsymbol{x}}_{k, n}=\hat{\boldsymbol{\Sigma}}_{k, n}\left(\boldsymbol{\Sigma}_{n}^{-1} \boldsymbol{\mu}_{n}+\boldsymbol{C}^{\mathrm{T}} \boldsymbol{R}_{k, n}^{-1}\left(\boldsymbol{y}_{k, n}-\boldsymbol{b}\right)\right) .
$$

The second derivative is the curvature of the quadratic function describing the posterior distribution [29. Chap. 3], whose inverse is the posterior covariance matrix

$$
\hat{\boldsymbol{\Sigma}}_{k, n}=\left(\boldsymbol{\Sigma}_{n}^{-1}+\boldsymbol{C}^{\mathrm{T}} \boldsymbol{R}_{k, n}^{-1} \boldsymbol{C}\right)^{-1} .
$$

The inference scheme is fully differentiable, which makes it possible to directly utilize Eqs. (3) and (4) for learning the previously described model parameters via back-propagation.

\subsection{Loss function}

A modified version of the SEL loss function introduced in [21] is utilized in this work, which additionally takes into account the probabilistic nature of the localization output stage. The probabilistic SEL loss is computed as

$$
\mathcal{L}_{n}=\mathcal{L}_{\mathrm{ACT}}\left(\hat{\gamma}_{n}, \gamma_{n}\right)+\alpha \mathcal{L}_{\mathrm{DOA}}\left(\hat{\boldsymbol{x}}_{n}, \boldsymbol{x}_{n}, \gamma_{n}\right)+\beta \mathcal{L}_{\mathrm{KL}}\left(\hat{\boldsymbol{\Sigma}}_{n}\right)
$$

for each of the $n=1, \ldots, N$ sound sources that can be represented by the model. The discrete time index $k$ is omitted here and in the following for notational convenience and $\alpha, \beta \in \mathbb{R}_{+}$are scaling factors. Eq. (5) incorporates a binary cross-entropy loss term $\mathcal{L}_{\mathrm{ACT}}\left(\hat{\gamma}_{n}, \gamma_{n}\right)$ between estimated and ground-truth source activities $\hat{\gamma}_{n}$ and $\gamma_{n}$, as well as a DoA loss term $\mathcal{L}_{\mathrm{DOA}}\left(\hat{\boldsymbol{x}}_{n}, \boldsymbol{x}_{n}, \gamma_{n}\right)=\xi_{n} \gamma_{n}$ utilizing the DoA error

$$
\xi_{n}=\operatorname{acos}\left(\sin \left(\hat{\phi}_{n}\right) \sin \left(\phi_{n}\right)+\cos \left(\hat{\phi}_{n}\right) \cos \left(\phi_{n}\right) \cos \left(\Delta \vartheta_{n}\right)\right),
$$

with $\Delta \vartheta_{n}=\vartheta_{n}-\hat{\vartheta}_{n}$. The term $\mathcal{L}_{\mathrm{KL}}\left(\hat{\boldsymbol{\Sigma}}_{n}\right)$ is required to prevent the system from setting the parameterized covariance matrices to zero. It models the Kullback-Leibler divergence (KLD) between $p\left(\boldsymbol{x}_{k, n} \mid \boldsymbol{y}_{k, n}\right)$ and a corresponding multivariate Gaussian distribution with identical mean and unit covariance matrix, similar to the penalty term utilized for training variational autoencoders $|30|$. The final loss is obtained by summing the individual losses in Eq. (5) over all time steps and sources, utilizing permutation invariant training [31].

\section{Evaluation}

The proposed PILOT framework is evaluated on three publicly available datasets. The detailed training procedure and experimental setup is described in the following. The program code is accessible online

\subsection{Datasets}

The ANSYN, RESYN and REAL datasets presented in [14] are used as evaluation corpora in this work. All three datasets are composed of multi-channel audio signals in first-order Ambisonics format. The first two datasets use simulated anechoic and reverberant impulse responses, whereas the latter one is based on impulse responses recorded in realistic environments. All datasets comprise three subsets each, corresponding to either no, at most two, or at most three temporally overlapping sources. Each subset provides three cross-validation splits with 300 audio files. These files are divided into 240 files for training and 60 for validation. The audio signals are sampled with $44.1 \mathrm{kHz}$ and have a duration of $30 \mathrm{~s}$ each. The audio signals in the synthetic datasets contain sound events from 11 different classes, covering the full azimuth range and an elevation range from $-60^{\circ}$ to $60^{\circ}$. The REAL dataset utilizes sound events from 8 classes. It covers the full azimuth range and the elevation range was restricted between $-40^{\circ}$ and $40^{\circ}$.

\subsection{Baseline methods}

Three baseline methods were employed in this work. The CNN feature extraction stage described in Sec. 3.1 is used as a first baseline to establish a lower bound on performance. Herein, all intermediate steps between the feature extraction and the output stage are omitted, yielding a small model with 110668 parameters. Additionally, the modified SELDNet architecture [14] (without event type association to guarantee a fair comparison) and the ADRENALINE framework described in [21] constitute the second and third baseline models with 551500 and 448204 parameters, respectively. Both architectures employ recurrent structures via gated recurrent units (GRUs) and the latter method additionally makes use of attention mechanisms. The proposed PILOT framework incorporates 466736 trainable parameters in total.

\subsection{Performance metrics}

Frame recall and DoA error are used as performance metrics. The frame recall metric [14] indicates the percentage of frames, where the number of active sources was estimated correctly. A source is considered active if its indicator variable in the source activity vector exceeds a threshold of 0.5. The DoA error on the test set is computed using the definition in Eq. (6). Herein, all active sources at a particular frame are incorporated and the

1 https://github.com/rub-ksv/pilot 
Hungarian algorithm [32] is used to solve the assignment problem between multiple estimated DoAs and the target DoAs.

\subsection{Experimental setup}

Both CNN and SELDNet baselines were parameterized as reported in [14]. The parameters of the ADRENALINE baseline model were also specified as outlined in the corresponding publication [21]. The loss term in Eq. (5) uses internal scaling factors of $\alpha=\beta=1$. The Gaussian prior in Eq. (1) was initialized with equally spaced azimuth angles and elevation set to zero for $\boldsymbol{\mu}_{n}$ and $\boldsymbol{\Sigma}_{n}=\boldsymbol{I}$ for $n=1, \ldots, N$. Kaiming initialization [33] was used to initialize the remaining parameters of all evaluated models. Training was conducted using the AdamW optimizer [34] with batch-size 256 and a learning rate of 0.05 . The learning rate was varied by adopting the scheduling scheme from [19]. Separate models were trained for 200 epochs in each cross-validation fold. The models yielding the best validation loss were chosen for performance evaluation on the test sets.

\section{Results and discussion}

The experimental results are depicted in Figs. 3 and 4 An initial application of the Shapiro-Wilk test [35] on the resulting DoA errors rejected the null-hypothesis of normally distributed samples. Hence, the one-sided Mann-Whitney- $U$ test [36] was used to assess statistically significant differences in DoA error between the proposed PILOT framework and the three baselines.

The results indicate that the proposed PILOT framework outperforms all three baseline methods in terms of DoA error with statistically significant differences in all evaluation scenarios. Notably, all methods that incorporate temporal context via either RNNs or transformers yield improved performance over the CNN baseline. However, it has to be taken into account here that the $\mathrm{CNN}$ baseline has significantly fewer parameters than the other models. A direct comparison between ADRENALINE and PILOT reveals that, even though both models have nearly the same capacity in terms of trainable parameters, the transformer-based PILOT framework consistently outperforms the RNN-based baseline model. This finding provides empirical evidence that transformers can be successfully applied to SEL tasks on the datasets evaluated in this study. Additionally, PILOT comes up with estimation uncertainties, which none of the other frameworks is able to provide, cf. Fig. 1]

In terms of frame recall, the proposed PILOT model yields significantly better results than the modified SELDNet and ADRENALINE baselines on the evaluated datasets. All frameworks show a similar drop in performance for estimating the number of sound events in reverberant environments. This indicates that reverberation entails a challenge for distinguishing between concurrent sound events, even though temporal context is explicitly utilized by the proposed PILOT system.

\section{Conclusion and outlook}

In summary, the results presented in this work provide a first promising perspective on how transformer-based models can be exploited for SEL tasks. The proposed PILOT framework shows that such models contribute to beneficial performance in SEL tasks compared to RNN-based systems. Moreover, a differentiable probabilistic localization output stage based on a linear Gaussian system was introduced that provides a means to estimate uncertainties for deep learning-based sound event localization. Future work might focus on incorporating these
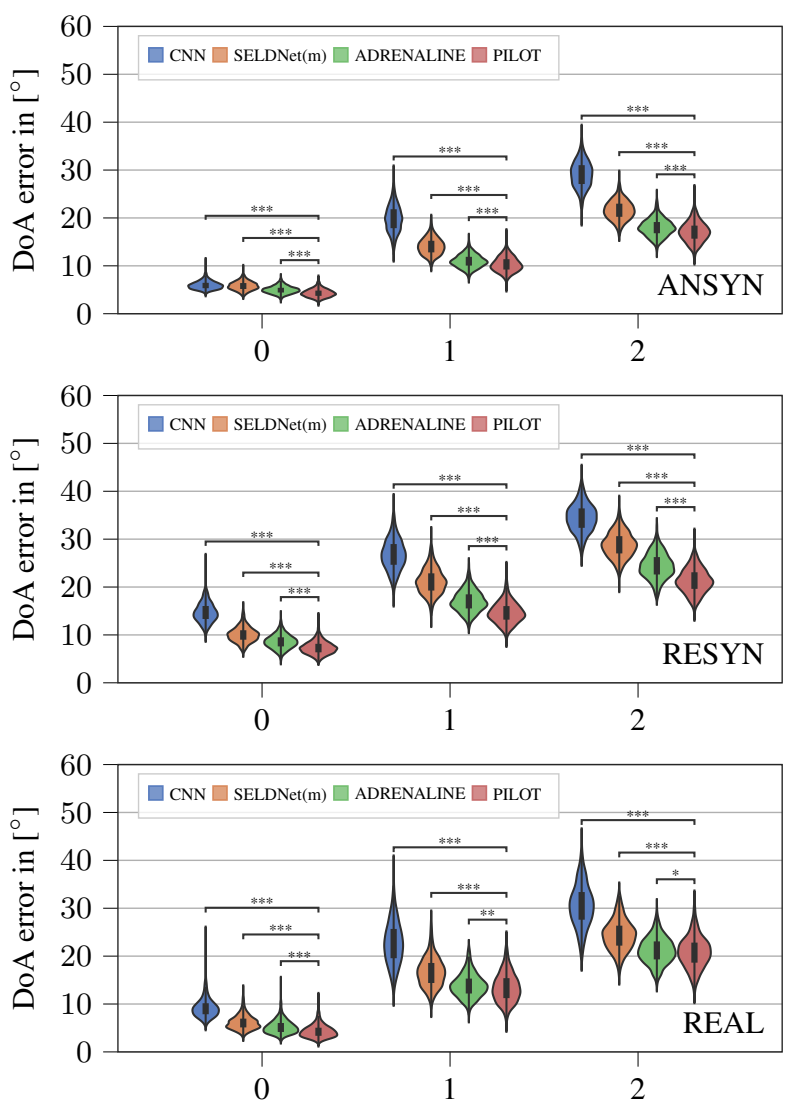

Max. number of temporally overlapping sources

Figure 3: Grouped violin-plots of DoA errors obtained over all cross-validation folds for the ANSYN, RESYN and REAL datasets. Three asterisks (***) indicate a statistically significant difference with $p<0.001$, two asterisks $(* *)$ correspond to $p<0.01$ and one asterisk $(*)$ denotes $p<0.05$.

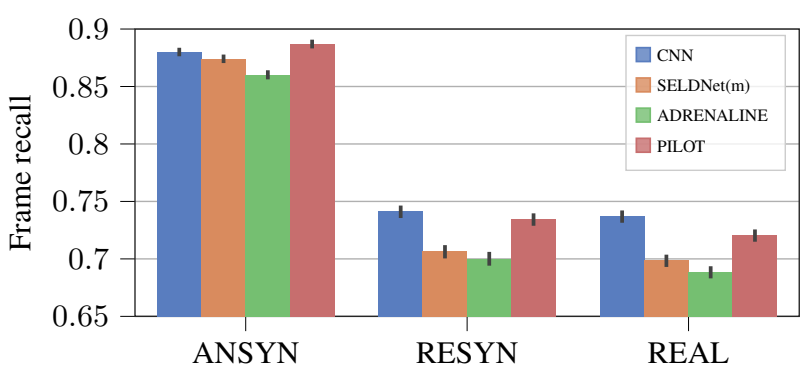

Figure 4: Frame recall of all investigated methods with standard deviations (denoted by the black bars), averaged over all crossvalidation folds.

uncertainties into suitable applications and evaluating their benefits to tasks related to, e.g., decision making.

\section{References}

[1] R. Li, D. Pan, and S. Zhang, "Speech enhancement algorithm based on sound source localization and scene matching for binaural digital hearing aids," Journal of Medical and Biological Engineering, vol. 39, pp. 403-417, Apr 2018. 
[2] F. Liu, Y. Yang, and Q. Lin, "Sound source localization and speech enhancement algorithm based on fixed beamforming," in Proceedings of the 2019 4th International Conference on Automation, Control and Robotics Engineering, ser. CACRE2019. New York, NY, USA: Association for Computing Machinery, 2019.

[3] S. Araki, M. Fujimoto, K. Ishizuka, H. Sawada, and S. Makino, "A DOA based speaker diarization system for real meetings," in 2008 Hands-Free Speech Communication and Microphone Arrays, 2008, pp. 29-32.

[4] J. Schmalenstroeer and R. Haeb-Umbach, "Online diarization of streaming audio-visual data for smart environments," IEEE Jour nal of Selected Topics in Signal Processing, vol. 4, no. 5, pp. 845856, 2010.

[5] M. Wölfel, K. Nickel, and J. McDonough, "Microphone array driven speech recognition: Influence of localization on the word error rate," in Machine Learning for Multimodal Interaction, S. Renals and S. Bengio, Eds. Berlin, Heidelberg: Springer Berlin Heidelberg, 2006, pp. 320-331.

[6] C. J. Grobler, C. P. Kruger, B. J. Silva, and G. P. Hancke, "Sound based localization and identification in industrial environments,' in 43rd Annual Conference of the IEEE Industrial Electronics Society, Oct 2017, pp. 6119-6124.

[7] K. Kodera, A. Itai, and H. Yasukawa, "Sound localization of approaching vehicles using uniform microphone array," in 2007 IEEE Intelligent Transportation Systems Conference, 2007, pp. 1054-1058

[8] R. Schmidt, "Multiple emitter location and signal parameter estimation," IEEE Transactions on Antennas and Propagation, vol. 34, no. 3, pp. 276-280, March 1986.

[9] J. H. DiBiase, H. F. Silverman, and M. S. Brandstein, Robust Lo calization in Reverberant Rooms. Berlin, Heidelberg: Springer, 2001, pp. 157-180

[10] H. Sawada, R. Mukai, S. Araki, and S. Makino, "Multiple source localization using independent component analysis," in 2005 IEEE Antennas and Propagation Society International Symposium, vol. 4B, July 2005, pp. 81-84 vol. 4B

[11] S. Chakrabarty and E. A. P. Habets, "Broadband DOA estimation using convolutional neural networks trained with noise signals," in 2017 IEEE Workshop on Applications of Signal Processing to Audio and Acoustics (WASPAA), Oct 2017, pp. 136-140.

[12] W. He, P. Motlicek, and J. Odobez, "Deep neural networks for multiple speaker detection and localization," in 2018 IEEE International Conference on Robotics and Automation (ICRA), May 2018, pp. 74-79.

[13] S. Adavanne, A. Politis, and T. Virtanen, "Direction of arrival estimation for multiple sound sources using convolutional recurren neural network," in 2018 26th European Signal Processing Conference (EUSIPCO), Sep. 2018, pp. 1462-1466.

[14] S. Adavanne, A. Politis, J. Nikunen, and T. Virtanen, "Sound event localization and detection of overlapping sources using convolutional recurrent neural networks," IEEE Journal of Selected Topics in Signal Processing, vol. 13, no. 1, pp. 34-48, March 2019.

[15] U. Klee, T. Gehrig, and J. McDonough, "Kalman filters for time delay of arrival-based source localization," EURASIP J. Adv. Signal Process, vol. 2006, p. 167, Jan. 2006.

[16] H. Hao, H. Liang, and Y. Liu, "Particle methods for real-time sound source localization based on the multiple signal classification algorithm," in 2014 International Conference on Intelligent Green Building and Smart Grid (IGBSG), 2014, pp. 1-5.

[17] S. Hochreiter and J. Schmidhuber, "Long short-term memory," Neural Computation, vol. 9, no. 8, pp. 1735-1780, 1997.

[18] T. H. Trinh, A. M. Dai, T. Luong, and Q. V. Le, "Learning longerterm dependencies in rnns with auxiliary losses," in ICML, 2018.

[19] A. Vaswani, N. Shazeer, N. Parmar, J. Uszkoreit, L. Jones, A. N. Gomez, L. Kaiser, and I. Polosukhin, "Attention is all you need," in Advances in Neural Information Processing Systems 30. Curran Associates, Inc., 2017, pp. 5998-6008.
[20] K. P. Murphy, Machine learning: A probabilistic perspective. Cambridge, Mass. [u.a.]: MIT Press, 2013.

[21] C. Schymura, T. Ochiai, M. Delcroix, K. Kinoshita, T. Nakatani, S. Araki, and D. Kolossa, "Exploiting attention-based sequenceto-sequence architectures for sound event localization," in 2020 28th European Signal Processing Conference (EUSIPCO), 2021, pp. 231-235.

[22] K. Cho, B. van Merriënboer, C. Gulcehre, D. Bahdanau, F. Bougares, H. Schwenk, and Y. Bengio, "Learning phrase representations using RNN encoder-decoder for statistical machine translation," in Proceedings of the 2014 Conference on Empirical Methods in Natural Language Processing (EMNLP). Doha, Qatar: Association for Computational Linguistics, Oct 2014, pp. 1724-1734.

[23] T. Haarnoja, A. Ajay, S. Levine, and P. Abbeel, "Backprop KF Learning discriminative deterministic state estimators," in Advances in Neural Information Processing Systems 29, 2016, pp. 4376-4384.

[24] R. Jonschkowski, D. Rastogi, and O. Brock, "Differentiable particle filters: End-to-end learning with algorithmic priors," in Proceedings of Robotics: Science and Systems, Pittsburgh, Pennsylvania, June 2018.

[25] P. Becker, H. Pandya, G. Gebhardt, C. Zhao, C. J. Taylor, and G. Neumann, "Recurrent Kalman networks: Factorized inference in high-dimensional deep feature spaces," in Proceedings of the 36th International Conference on Machine Learning, ICML 2019, 9-15 June 2019, Long Beach, CA, USA, ser. Proceedings of Machine Learning Research, vol. 97, 2019, p. 544-552.

[26] C. Schymura, T. Ochiai, M. Delcroix, K. Kinoshita, T. Nakatani, S. Araki, and D. Kolossa, "A dynamic stream weight backprop Kalman filter for audiovisual speaker tracking," in ICASSP 2020 - 2020 IEEE International Conference on Acoustics, Speech and Signal Processing (ICASSP), 2020, pp. 581-585.

[27] A. Deleforge, F. Forbes, and R. Horaud, "Acoustic space learning for sound-source separation and localization on binaural manifolds," International Journal of Neural Systems, vol. 25, no. 01, p. $1440003,2015$.

[28] S. Braun and I. Tashev, "Acoustic localization using spatial probability in noisy and reverberant environments," in Workshop on Applications of Signal Processing to Audio and Acoustics (WAS$P A A)$, IEEE. IEEE, October 2019, pp. 1-5.

[29] S. Thrun, W. Burgard, and D. Fox, Probabilistic Robotics. The MIT Press, 2005.

[30] D. P. Kingma and M. Welling, "Auto-encoding variational Bayes," in 2nd International Conference on Learning Representations, ICLR 2014, Banff, AB, Canada, April 14-16, 2014, Conference Track Proceedings, Y. Bengio and Y. LeCun, Eds., 2014.

[31] D. Yu, M. Kolbæk, Z. Tan, and J. Jensen, "Permutation invariant training of deep models for speaker-independent multi-talker speech separation," in 2017 IEEE International Conference on Acoustics, Speech and Signal Processing (ICASSP), 2017, pp. 241-245.

[32] H. W. Kuhn and B. Yaw, "The Hungarian method for the assignment problem," Naval Res. Logist. Quart, pp. 83-97, 1955.

[33] K. He, X. Zhang, S. Ren, and J. Sun, "Delving deep into rectifiers: Surpassing human-level performance on imagenet classification," in 2015 IEEE International Conference on Computer Vision (ICCV), Dec 2015, pp. 1026-1034.

[34] I. Loshchilov and F. Hutter, "Decoupled weight decay regularization," in International Conference on Learning Representations, 2019.

[35] S. S. Shapiro and M. B. Wilk, "An analysis of variance test for normality (complete samples)," Biometrika, vol. 52, no. 3/4, pp. 591-611, Dec. 1965.

[36] H. B. Mann and D. R. Whitney, "On a test of whether one of two random variables is stochastically larger than the other," Annals of Mathematical Statistics, vol. 18, no. 1, pp. 50-60, 1947. 